\title{
Deficiência de ferro na gestação, parto e puerpério
}

\section{The iron deficiency in pregnancy, labor and puerperium}

Lilian P. Rodrigues ${ }^{l}$

Silvia Regina P. F. Jorge $e^{2}$

\begin{abstract}
A anemia por deficiência de ferro representa desordem nutricional de maior prevalência em todo o mundo. As duas causas mais comuns de anemia na gestação e pós-parto são a deficiência de ferro e as perdas sanguineas agudas. Na gestação, as mudanças fisiológicas podem dificultar o diagnóstico das doenças hematológicas. O propósito deste estudo é revisar a deficiência de ferro na gestação, as consequências adversas materno-fetais, o diagnóstico e manejo da anemia na gestação. Rev. Bras. Hematol. Hemoter. 2010;32(Supl.2):53-56.
\end{abstract}

Palavras-chave: Anemia; deficiência de ferro; gestação.

\section{Introdução}

A gravidez impõe adaptações ao organismo materno. Ajustes fisiológicos são necessários para que ocorra o adequado desenvolvimento do produto conceptual. Muitas dessas mudanças iniciam-se precocemente e se estendem por toda gestação até o término da lactação.

\section{Anemia fisiológica da gravidez}

O volume sanguíneo começa a aumentar desde o primeiro trimestre, por ação de hormônios - estrogênio e progesterona - e sob a influência do sistema renina-angiotensina-aldosterona. Atinge platô ao redor da $30^{\mathrm{a}}$ semana, com aumento de $50 \%(1.200 \mathrm{~mL}$ a $1.500 \mathrm{~mL})$ em relação ao encontrado no período pré-conceptual. Consequentemente, o débito cardíaco se eleva em $40 \%$ a $50 \%$. Ocorre aumento da massa eritrocitária em menores proporções, $20 \%$ a $30 \%$ $(300 \mathrm{~mL})$ principalmente em resposta à atividade aumentada da eritropoetina e hormônio lactogênio placentário, que estimulam a eritropoese medular (hiperplasia medular eritroide). Para acomodação desta expansão volumétrica, a resistência vascular periférica diminui. ${ }^{1,2}$
Estas adaptações gravídicas são possíveis não só pelo aumento da taxa de absorção do ferro como também pelo aumento da transferrina circulante devido ao estímulo estrogênico. Contudo, apesar de todos estes mecanismos compensatórios, o aumento do volume eritrocitário é desproporcional ao aumento do volume plasmático, ocorrendo gradativamente queda do hematócrito, hemoglobina e viscosidade sanguínea, conduzindo a um estado de hemodiluição (anemia fissiológica da gravidez).

As menores concentrações de hemoglobina circulante, decorrente das adaptações gravídicas, não causam prejuízo para o binômio materno-fetal. Nesta condição, a perfusão e oxigenação tecidual são mantidas às custas do estado hipervolêmico.

\section{Conceito}

A Organização Mundial de Saúde (OMS) estabelece o limite aceitável de $11,0 \mathrm{~g} / \mathrm{dL}$ para concentrações de hemoglobina, abaixo do qual se define a anemia. ${ }^{3}$

Prevalência

Estima-se que a anemia não fisiológica acomete $20 \%$ a $80 \%$ \% das gestações. Destas, a anemia por deficiência de

${ }^{1}$ Ginecologista e obstetra. Professora Assistente da Faculdade de Ciências Médicas da Santa Casa de São Paulo - São Paulo-SP.

${ }^{2}$ Ginecologista e obstetra. Professora Instrutora da Faculdade de Ciências Médicas da Santa Casa de São Paulo- São Paulo-SP.

Faculdade de Ciências Médicas da Santa Casa de São Paulo. Setor de Gestação de Alto Risco do Departamento de Obstetrícia e Ginecologia da Irmandade da Santa Casa de Misericórdia de São Paulo - São Paulo-SP.

Correspondência: Lilian de Paiva Rodrigues

Rua Dr. Cesário Mota Jr, 112 - Vila Buarque

01221-020 - São Paulo-SP - Brasil

Tel.: (55 11) 3222-4254; Fax: (55 11) 2176-7384

E-mail:cvk@uol.com.br

Doi: 10.1590/S1516-84842010005000057 
ferro é a mais comum desordem nutricional encontrada. Estima-se que aproximadamente $50 \%$ das grávidas em todo o mundo são anêmicas, sendo que $52 \%$ encontram-se em países não industrializados e $23 \%$ em países industrializados. $^{4}$

Entre os segmentos biológicos mais vulneráveis ao problema acham-se as mulheres no período reprodutivo, particularmente durante a gestação. ${ }^{5}$

\section{Etiologia}

Do ponto de vista etiológico, a anemia poderá ocorrer devido à perda sanguínea, destruição excessiva de eritrócitos ou deficiência de sua produção. ${ }^{6}$ Neste último grupo encontramos a anemia por deficiência de ferro sendo o tipo mais frequente e preocupante do ponto de vista de saúde pública

\section{Repercussões clínicas maternas e fetais}

A depender do grau de anemia, a sua associação à gestação pode ocasionar efeitos deletérios ao binômio maternofetal. Aproximadamente $40 \%$ das mortes maternas e perinatais são ligadas à anemia. ${ }^{4}$

As principais repercussões maternas são: comprometimento do desempenho físico e mental, labilidade emocional, pré-eclâmpsia, alterações cardiovasculares, diminuição da função imunológica, alterações da função da tireoide e catecolaminas, queda de cabelos, enfraquecimento das unhas. ${ }^{7,89}$ Outro aspecto a ser considerado é a menor tolerabilidade às perdas sanguíneas do parto, conduzindo a maior risco de anemia pós-parto e hemotransfusão. ${ }^{10}$

Em relação ao comprometimento fetal, o estado anêmico relaciona-se com perdas gestacionais (abortamentos, óbito intrauterino), hipoxemia fetal, prematuridade, ruptura prematura das membranas ovulares, quadros infecciosos, restrição de crescimento fetal, e muitas vezes com alterações irreversíveis do desenvolvimento neurológico fetal. ${ }^{10}$

Valores de hemoglobina materna inferiores a $6,5 \mathrm{~g} / \mathrm{dL}$ associam-se a comprometimento da vitalidade fetal e aumento da mortalidade perinatal. ${ }^{10}$

\section{Abordagem diagnóstica}

O rastreamento para anemia deve ser realizado de forma universal nas gestantes, por ocasião do acompanhamento pré-natal. Avaliação clínica adequada, com investigação detalhada dos sintomas apresentados e minucioso exame físico são elementos úteis para determinar a gravidade da doença, bem como orientar o tratamento. Nos quadros leves, sintomas como mal-estar, cansaço, fadiga podem se confundir aos achados da gestação normal. Taquicardia, palidez cutânea, dispneia aos esforços, ou mesmo ao repouso, sugerem anemias moderadas ou severas. A quantificação dos índices hematimétricos apresenta valor limitado para o diagnóstico da anemia ferropriva. $\mathrm{Na}$ gestação, o volume corpuscular médio (VCM) aumenta, discretamente durante os dois primeiros trimestres, podendo mascarar uma deficiência de ferro. Testes para avaliar o perfil do ferro no organismo, dosagem do ferro sérico, a capacidade total de ligação de ferro, saturação da transferrina sofrem flutuações decorrentes de mecanismos adaptativos próprios da gestação. Assim, a ferritina sérica é considerada o melhor teste laboratorial para avaliar a quantidade de ferro disponível, por estar diretamente relacionada ao seu depósitos orgânico.

Na Tabela 1encontramos os valores dos testes utilizados para o diagnóstico de anemia ferropriva na gestação. ${ }^{11}$

Tabela 1. Valores laboratoriais encontrados na anemia ferropriva em gestantes

\begin{tabular}{ll}
\hline Exame & \multicolumn{1}{c}{ Valor I } \\
\hline Hemoglobina & $<11 \mathrm{~g} / \mathrm{dL}$ \\
Ferritina sérica & $<12 \mathrm{ng} / \mathrm{mL}$ \\
Capacidade total de ligação do ferro & $>400 \mathrm{mcg} / \mathrm{dL}$ \\
Ferro plasmático & $<50 \mathrm{mcg} / \mathrm{dL}$ \\
Índice de saturação da transferrina & $<16 \%$ \\
Volume corpuscular médio $(\mathrm{VCM})$ & Diminuído \\
Hemoglobina corpuscular média $(\mathrm{HCM})$ & Diminuída \\
\hline
\end{tabular}

\section{Prevenção}

Apesar das evidências de que grande proporção de mulheres na idade reprodutiva engravida com baixas reservas de ferro, ${ }^{12}$ a necessidade de suplementação de ferro de rotina nesse período ainda é controversa. ${ }^{13}$

Estudos têm demonstrado que, em condições normais, gestantes não suplementadas apresentam valores de hemoglobina, hematócrito e ferritina diminuídos durante a gestação, principalmente a partir do segundo trimestre. ${ }^{14,15}$ Apesar da redução da hemoglobina ser fisiológica no segundo trimestre da gestação, a queda dos estoques de ferro, refletidas pelos valores da ferritina, justificam a utilização de ferro suplementar.

Assim, a OMS recomenda, de modo geral, suplementação com $60 \mathrm{mg}$ de ferro elementar para toda gestante a partir da $20^{\text {a }}$ semana de gravidez, ${ }^{1,16-20}$ o que corresponde à suplementação com $300 \mathrm{mg}$ de sulfato ferroso, visto que a quantidade de ferro elementar corresponde a $20 \%$ da dose total do sal.

A orientação nutricional deve ser realizada durante todo acompanhamento, de preferência já no período antenatal. $\mathrm{O}$ ferro proveniente da dieta pode ser de origem vegetal, ferro não heme, que tem baixa absorção, ao redor de $1 \%$ a $7 \%$, contribuindo muito pouco para as reservas maternas; e o ferro de origem animal, ferro heme, cuja taxa de absorção 
varia entre $20 \%$ a $30 \%$, com boa disponibilidade, e que definitivamente contribui para o estoque materno. Assim, recomenda-se o consumo de carnes em geral, evitar associação com cereais integrais, uso de frutas ricas em vitamina C, entre outras medidas. ${ }^{10,16,17,21}$

Nos casos de anemia ferropriva na gestação, além da identificação e correção da causa quando possível, orientação dietética, recomenda-se terapia com reposição de ferro elementar.

A terapia por via oral, via de regra, é a primeira opção de tratamento. Recomenda-se dose de $120 \mathrm{mg}$ a $180 \mathrm{mg}$ de ferro elementar ( $2 \mathrm{mg}$ a $5 \mathrm{mg} / \mathrm{kg} / \mathrm{dia}$ ), administrada em duas ou três tomadas, preferencialmente uma hora antes das refeições principais. A resposta ao tratamento é considerada adequada quando se constata incremento de $50 \%$ ou mais dos valores iniciais da hemoglobina após trinta dias de administração, e o tempo de tratamento para reposição das reservas maternas depende da intensidade da deficiência de ferro e correção da causa da anemia. Contudo, a efetividade do tratamento depende da capacidade de absorção intestinal, e, principalmente, da tolerância ao tratamento oral, acompanhado por efeitos colaterais, principalmente gastrointestinais (náuseas, vômitos, epigastralgia, diarreia e constipação intestinal). Em aproximadamente $10 \%$ a $40 \%$ dos pacientes, a intolerância é tão intensa que inviabiliza o tratamento por via oral. ${ }^{1,3,16,18,20}$

A terapia com ferro parenteral constitui-se em alternativa eficaz para os casos de intolerância ao tratamento oral, quer seja por dificuldade de ingestão ou absorção, onde a reposição oral mostra-se insuficiente para suprir as necessidades orgânicas, ou, ainda, em situações em que a deficiência de ferro é grave (hemoglobina inferior a 7,0 $\mathrm{gr} / \mathrm{dL}$ ), após a $14^{\mathrm{a}}$ semana, e quando se pretende resposta mais rápida para diminuir as chances de transfusão de hemácias. ${ }^{22}$

A transfusão de hemácias é indicada mais raramente no período pré-natal, porém, nos casos de anemia intensa, grave, com hemoglobina menor que $7,0 \mathrm{~g} / \mathrm{dL}$, com descompensação materna, associação com outras doenças, prejuízo da vitalidade fetal, risco de procedimento cirúrgico ou parto emergencial, tal procedimento se justifica para prevenção das complicações maternas fetais provenientes da anemia. ${ }^{21,22}$

\section{Deficiência de ferro no parto}

Por ocasião do parto não complicado, ocorrem perdas sanguíneas maternas estimadas entre $500 \mathrm{~mL}$ a $1.000 \mathrm{~mL}$ nos partos vaginais e cesáreos respectivamente. ${ }^{1}$

Na gestante anêmica, esta perda, considerada fisiológica, proveniente da dequitação, contratilidade uterina e lacerações de tecidos maternos, resulta em uma sobrecarga maior, transformando-se em processo espoliativo.

Idealmente, como já citado, a correção da anemia ferropriva deve ocorrer no período pré-concepcional ou pré- natal, preferentemente por via oral. Quando não possível, por motivo de intolerância absoluta ou má absorção, ou, ainda, mediante a proximidade do parto, a reposição por via parenteral intravenosa é opção eficaz e segura. Na vigência de instabilidade hemodinâmica, anemia grave (hemoglobina menor que $7,0 \mathrm{~g} / \mathrm{dL}$ ) e imediatismo do parto, a transfusão de hemácias deve ser indicada. ${ }^{21,22}$

\section{Deficiência de ferro no puerpério e lactação}

Ainda, no período puerperal imediato ocorrem perdas sanguíneas (loquiação), que, em condições normais, gradativamente vão diminuindo, à medida que a contratilidade e involução uterina se estabelecem, bem como a cicatrização de tecidos que participam do canal de parto, eventualmente lacerados por ocasião do nascimento. Do mesmo modo, este processo fisiológico pode se transformar em processo espoliativo, e a reposição de ferro está indicada.

Especial cuidado deve ser tomado nesta fase quanto às perdas sanguíneas aumentadas. A atonia uterina, decorrente da hipocontratilidade da fibra miometrial, frequentemente conduz a quadros de sangramento agudo, não raro choque hipovolêmico e morte materna. Neste caso, tratamento de suporte à vida da puérpera (correção do choque hipovolêmico, distúrbio de coagulação e metabólico, e prática de manobras de indução à contração uterina) deve ser prontamente instituído, além da correção da anemia

A lactação resulta em liberação de pequena quantidade de ferro pelo leite; contudo, o ferro necessário para manter seis meses de aleitamento exclusivo é equivalente a aproximadamente $14 \%$ do estoque materno corporal e representa metade do que é perdido comumente na menstruação. Assim, a necessidade de ferro durante a lactação é reduzida em 50\% enquanto a mulher está em amenorreia $(0,1 \mathrm{mg}$ a $0,3 \mathrm{mg}$ de ferro adicional por dia, compensada pela ausência de menstruação). Quando a menstruação retorna, as reservas de ferro corporais materno podem diminuir drasticamente se a ingestão do mineral for baixa. ${ }^{17}$

\begin{abstract}
Iron-deficiency anemia is a highly prevalent nutritional disorder in the whole world. The two most common causes of anemia in pregnancy and in the puerperium are iron deficiency and acute blood loss. Pregnancy is associated with physiological changes that may complicate the diagnosis of hematologic diseases. The purpose of this paper is to provide a brief overview of iron deficiency in pregnancy, adverse maternal-fetal consequences, screening and clinical management of anemia during pregnancy. Rev. Bras. Hematol. Hemoter. 2010;32(Supl.2):53-56.
\end{abstract}

Key words: Anemia; iron deficiency; pregnancy. 


\section{Referências Bibliográficas}

1. Grewal M, Biswas MK, FACOG, FRCOG, Dorothee P. Distúrbios cardíacos, hematológicos, pulmonares, renais e urinários na gravidez. In: DeCherney $\mathrm{AH}$, Nathan L. Current obstetrícia e ginecologia: diagnóstico e tratamento. $9^{\mathrm{a}}$ ed. São Paulo: McGraw Hill; 2005. p. 325-58.

2. Sifakis S, Pharmakides G. Anemia in pregnancy. Ann N Y Acad Sci. 2000;900:125-36.

3. World Health Organization. Nutritional anemias. Report of a WHO Scientific Group. Technical Report Series n 405. Genebra; 1968.

4. World Health Organization. Iron deficiency anaemia: assessment, prevention and control. A guide for programme managers. Geneva: World Health Organization; 2001. (WHO/NHD/01.3)

5. Batista Filho M, Ferreira LOC. Prevenção e tratamento da anemia nutricional ferropriva: novos enfoques e perspectivas. Cad. Saúde Pública. 1996;12:411-5.

6. Wintrobe MM, Lukens JN, Lee GR. The approach to the patient with anemia. In: Lee GR, Bithell TC, Foerster J, Athens JW, Lukens JN. Wintrobes clinical hematology. $9^{\text {th }}$ ed. Phildelphia: Lea \& Febiger; 1993. v.1, p. 715-44.

7. Van den Broek N. Anaemia in pregnancy in developing countries. Br J Obstet Gynaecol. 1998;105(4):385-90.

8. Baker WF Jr. Iron deficiency in pregnancy, obstetrics, and gynecology. Hematol Oncol Clin North Am. 2000;14(5):1061-77.

9. Hercberg S, Galan P, Preziosi P, Aissa M. Consequences of iron deficiency in pregnant women: current issues. Clin Drug Invest. 2000;19(suppl. 1):1-7.

10. Milman N. Prepartum aneamia: prevention and treatament. Ann Hematol.2008;87(12):949-59.

11. Cançado RD. Anemias. In: Sebastião Piato. Complicações em obstetrícia. São Paulo: Manole; 2009. p.485-512.

12. Lopes MCS, Ferreira LOC, Batista Filho M. Uso diário e semanal de sulfato ferroso no tratamento de anemia em mulheres no período reprodutivo. Cad Saúde Pública. 1999;15:799-808.

13. Beard JL. Effectiveness and strategies of iron supplementation during pregnancy. Am J Clin Nutr. 2000;71(5 Suppl):1288S-94S.

14. Andrade GN, Araújo DAC, Andrade ATL. Alterações dos estoques de ferro durante a gestação na mulher brasileira pela medida da ferritina sérica. J Bras Ginecol. 1996;106:345-9
15. Petraglia B, Silva LGP, Rezende Filho J, Chaves Netto H, Montenegro CAB. Avaliação dos valores eritrocitários no ciclo grávido-puerperal. J Bras Ginecol. 1994;104:139-44.

16. American College of Obstetricians and Gynecologists. ACOG Practice Bulletin $\mathrm{N}^{\circ}$ 95: anemia in pregnancy. Obstet Gynecol. 2008;112:201-7.

17. Institute of Medicine. Food and Nutrition Board. Dietary reference intakes for vitmin A, vitamin $\mathrm{K}$, arsenic, boron, chromium, iodine, iron, manganese, molybdenum, nickel, silicon, copper, iovanadium and zinc. Washington, D.C: National Academy Press; 2001.

18. Brasil. Ministério da Saúde. Assistência pré-natal: manual técnico. 3a ed. Brasília, D.F.: Ministério da Saúde; 2000. 60p.

19. 19. Brasil. Ministério da Saúde. Assistência pré-natal: manual técnico. $3^{\mathrm{a}}$ ed. Brasília, D.F.: Ministério da Saúde; 2000. 60p.

20. Brasil. Ministério da Saúde. Secretaria de Políticas de Saúde. Gestação de alto risco: manual técnico. $3^{\mathrm{a}}$ ed. Brasília, D.F.: Ministério da Saúde; 2000. p.105-7.

21. Cançado RD. Anemia ferropriva: experiência com uso de sacarato de hidróxido de ferro III intravenoso no tratamento de pacientes adultos que não obtiveram resposta satisfatória com ferro oral. Médico Reporter. 2004; 57:54-6.

22. Breymann C. Treatment of iron deficiency anemia in pregnancy and postpartum with special focus on intravenous iron sucrose complex. J Med Assoc Thai. 2005; 88: (suppl.2):S108-S109.

O tema foi sugerido e avaliado pelo coeditor deste fascículo educativo, Rodolfo Delfinii Cançado, e pelo board interno da RBHH, e publicado após a concordância do editor, Milton Artur Ruiz.

Conflito de interesse: sem conflito de interesse

Recebido: 21/12/2009

Aceito: 16/01/2010 